\title{
Transplante cardíaco: a experiência do Instituto do Coração de Pernambuco com 35 casos
}

\author{
Fernando MORAES NETO*, Deuzeny TENÓRIO*, Claudio A. GOMES*, Euclides TENÓRIO*, Sheila \\ HAZIN*, Marcos MAGALHÃES*, Carlos R. MORAES*
}

RBCCV 44205-542

\begin{abstract}
Moraes Neto F, Tenório D, Gomes C A, Tenório E, Hazin S, Magalhães M, Moraes C R - Transplante cardíaco: a experiência do Instituto do Coração de Pernambuco com 35 casos. Rev Bras Cir Cardiovasc $2001 ; 16(2): 152-9$.
\end{abstract}

RESUMO: Casuística e Métodos: Entre agosto de 1991 e fevereiro de 2000, 35 transplantes cardíacos ortotópicos foram realizados no Instituto do Coração de Pernambuco. Vinte e nove pacientes eram do sexo masculino e 6 do feminino, variando a idade de 15 a 69 anos (média 46,9). O diagnóstico dos receptores foi miocardiopatia isquêmica em 18 pacientes, miocardiopatia dilatada idiopática em 13, doença mixomatosa em 2, doença valvar reumática em 1 e doença de Chagas em 1. Todos estavam em fase final da insuficiência cardíaca (classe funcional III ou IV da classificação da New York Heart Association), e a fração de ejeção variou de 16 a 27\% (média: 20,9 $\pm 2,9$ ). Em todos utilizou-se a clássica técnica cirúrgica descrita por Lower e Shumway, em 1960. O tempo médio de isquemia do enxerto foi de 91 \pm 21 minutos (variou de 60 a $180 \mathrm{~min}$.).

Resultados: Ocorreram 7 (20\%) óbitos no pós-operatório imediato. Treze outros pacientes apresentaram complicações não fatais, incluindo 5 episódios de rejeição. $O$ tempo médio de seguimento dos 28 sobreviventes foi de 31,2 meses (variou de 1 a 68 meses). Houve 14 mortes tardias devido a rejeição (4), infecção (4), doença arterial coronária (2), insuficiência renal crônica (2) e morte súbita (2). A curva actuarial estima sobrevida de $70 \%$ com 1 ano e de $30 \%$ aos 5 anos.

Conclusão: É possível manter um programa de transplante cardíaco em nossa comunidade, embora se deva esperar resultados piores do que os da experiência internacional devido às limitações sociais da população de receptores.

DESCRITORES: Transplante de coração. Cardiopatias, cirurgia.

\section{INTRODUÇÃO}

O transplante de coração é atualmente um procedimento eficiente no tratamento de pacientes em insuficiência cardíaca terminal. Entretanto, problemas de ordem logística, especialmente a escassez de doadores, impedem que o número de transplantes atenda a demanda existente. Essa realidade é mais evidente em países em desenvolvimento, como o Brasil, onde um número relativamente pequeno de centros tem conseguido manter, em atividade, programas de transplante cardíaco (1). Em 1991, instituímos em Recife um programa de transplante de coração (2), sendo objetivo deste trabalho relatar os resultados dos primeiros 35 casos operados.

\footnotetext{
Trabalho realizado no Instituto do Coração de Pernambuco - Real Hospital Português de Beneficência em Pernambuco. Recife, PE, Brasil. Recebido para publicação em agosto de 2000.

*Do Instituto do Coração de Pernambuco.

Endereço para correspondência: Fernando Moraes Neto. Instituto do Coração de Pernambuco. Av. Portugal, 163. Recife, PE. CEP: 52010-010. Telefax (81) 221-0382.e-mail:f_moraes@interway.com.br
} 
Moraes Neto F, Tenório D, Gomes C A, Tenório E, Hazin S, Magalhães M, Moraes C R - Transplante cardíaco: a experiência do Instituto do Coração de Pernambuco com 35 casos. Rev Bras Cir Cardiovasc 2001; 16(2): 152-9.

\section{CASUÍSTICA E MÉTODOS}

No período compreendido entre agosto de 1991 e fevereiro de 2000, 35 pacientes foram submetidos a transplante cardíaco no Instituto do Coração do Real Hospital Português de Beneficência em Pernambuco. Vinte e nove $(82,9 \%)$ eram do sexo masculino e $6(17,1 \%)$ do feminino, variando a idade entre 15 e 69 anos (média $=46,9$ anos; DP $=13,6$ ).

$\mathrm{Na}$ Tabela 1 observam-se os principais dados pré-operatórios desse grupo de doentes. Dezoito eram portadores de miocardiopatia isquêmica, 13 tinham miocardiopatia dilatada idiopática, 2 apresentavam doença mixomatosa, 1 tinha valvopatia reumática e 1 exibia miocardiopatia chagásica. Trinta $(85,7 \%)$ estavam em classe funcional IV, e 5 $(14,3 \%)$ na classe III, de acordo com os critérios da New York Heart Association. A fração de ejeção $(\mathrm{FE})$ variou de 16 a $27 \%$ (média $=20,9 \%$; $D P=2,9$ ), e a resistência vascular pulmonar oscilou entre 1,5 a 6 unidades Wood (média $=3,8 ; \mathrm{DP}=0,9$ ). Dezessete $(48,5 \%)$ pacientes haviam sido submetidos a 20 operações cardíacas prévias. Vinte e seis $(74,2 \%)$ necessitaram de internações prévias para

TABELA 1

\begin{tabular}{|c|c|c|c|c|c|c|c|}
\hline \multicolumn{8}{|c|}{ DADOS PRÉ-OPERATÓRIOS } \\
\hline CASO & ETIOLOGIA & $\begin{array}{c}\text { CLASSE } \\
\text { FUNCIONAL } \\
\text { NYHA }\end{array}$ & $\begin{array}{c}\text { FE } \\
\%\end{array}$ & $\begin{array}{c}\text { RVP } \\
\text { UNID. WOOD }\end{array}$ & $\begin{array}{l}\text { OPERAÇÕES } \\
\text { PRÉVIAS }\end{array}$ & INOTRÓPICOS & $\begin{array}{l}\text { OUTROS } \\
\text { DADOS }\end{array}$ \\
\hline 1 & Isquêmica & IV & 22 & 4.8 & - & Sim & Caquexia grave \\
\hline 2 & Idiopática & IV & 20 & 3.6 & - & Sim & -- \\
\hline 3 & Isquêmica & IV & 22 & 4.0 & - & Sim & -- \\
\hline 4 & Isquêmica & IV & 24 & 4.6 & - & Sim & -- \\
\hline 5 & Idiopática & IV & 18 & 3.7 & - & Sim & $\begin{array}{c}\text { Caquexia + } \\
\text { Escara + } \\
\text { Hemodiálise }\end{array}$ \\
\hline 6 & Isquêmica & IV & 21 & 4.8 & - & - & - \\
\hline 7 & Mixomatosa & IV & 16 & 3.8 & Troca mitral & Sim & -- \\
\hline 8 & Idiopática & IV & 18 & 4.3 & - & Sim & -- \\
\hline 9 & Isquêmica & IV & 26 & 2.6 & Rev. Miocárdica & - & Obesidade \\
\hline 10 & Isquêmica & IV & 24 & 3.6 & - & Sim & -- \\
\hline 11 & D. Chagas & IV & 18 & 4.2 & - & Sim & - \\
\hline 12 & Isquêmica & III & 24 & 2.8 & - & - & -- \\
\hline 13 & Isquêmica & III & 25 & 3.6 & Rev. Miocárdica & - & -- \\
\hline 14 & Idiopática & IV & 22 & 4.0 & Correção de CIA & Sim & -- \\
\hline 15 & Isquêmica & IV & 20 & 2.8 & Aneurismectomia VE & - & -- \\
\hline 16 & Isquêmica & III & 21 & 1.8 & Rev. Miocárdica & - & -- \\
\hline 17 & Idiopática & IV & 22 & 4.2 & - & Sim & -- \\
\hline 18 & Idiopática & IV & 16 & 3.8 & - & - & -- \\
\hline 19 & Reumática & IV & 20 & 4.4 & Troca mitral (X3) & Sim & - \\
\hline 20 & Idiopática & IV & 21 & 3.2 & - & Sim & -- \\
\hline 21 & Idiopática & IV & 19 & 4.4 & - & Sim & -- \\
\hline 22 & Idiopática & IV & 21 & 2.8 & - & Sim & 一- \\
\hline 23 & Isquêmica & III & 25 & 3.4 & Rev. Miocárdica & - & - \\
\hline 24 & Isquêmica & IV & 20 & 4.6 & Rev. Miocárdica & Sim & 一- \\
\hline 25 & Isquêmica & IV & 19 & 5.0 & Rev. Miocárdica & Sim & -- \\
\hline 26 & Idiopática & IV & 20 & 4.0 & - & Sim & 一- \\
\hline 27 & Idiopática & IV & 16 & 5.2 & Cirurgia para EMF & Sim & Anasarca \\
\hline 28 & Isquêmica & IV & 23 & 3.8 & Rev. Miocárdica & Sim & 一- \\
\hline 29 & Isquêmica & III & 27 & 2.8 & Rev. Miocárdica & - & Diabetes \\
\hline 30 & Isquêmica & IV & 22 & 4.4 & Rev. Miocárdica & Sim & Diabetes \\
\hline 31 & Idiopática & IV & 17 & 3.6 & - & Sim & Diabetes \\
\hline 32 & Mixomatosa & IV & 16 & 6.0 & Troca mitral & Sim & 一- \\
\hline 33 & Idiopática & IV & 23 & 3.9 & - & Sim & -- \\
\hline 34 & Isquêmica & IV & 22 & 4.0 & Rev. Miocárdica & Sim & -- \\
\hline 35 & Isquêmica & IV & 20 & 1.5 & Rev. Miocárdica (X2) & Sim & -- \\
\hline
\end{tabular}

RVP = Resistência Vascular Pulmonar; EMF = Endomiocardiofibrose; NYHA = New York Heart Association; FE = Fração de Ejeção. 
Moraes Neto F, Tenório D, Gomes C A, Tenório E, Hazin S, Magalhães M, Moraes C R - Transplante cardíaco: a experiência do Instituto do Coração de Pernambuco com 35 casos. Rev Bras Cir Cardiovasc 2001; 16(2): 152-9.

uso de inotrópicos ou estavam internados com suporte inotrópico à época do transplante. Três doentes (Casos 1, 5 e 27) exibiam situação clínica extremamente precária ao serem levados à sala de operações, um dos quais tinha falência múltipla de órgãos, estando há 20 dias em hemodiálise e com grande escara sacral.

Extensa avaliação clínica, laboratorial, psicológica e social foi realizada em todos os casos, e os critérios de seleção dos receptores foram os rotineiramente aceitos no presente (3)

Nos doadores, o diagnóstico clínico de morte cerebral foi confirmado por arteriografia e/ou eletroencefalografia. $O$ coração foi retirado no mesmo hospital em 33 casos e transportado a distância em dois casos.

A avaliação imunológica pré-operatória consistiu na pesquisa de anticorpos pelo painel reativo linfocitário e pelo "cross-matching" (compatibilidade entre linfócitos do doador e soro do receptor).

A operação foi realizada através esternotomia mediana com circulação extracorpórea convencional e hipotermia sistêmica moderada 24 - 28으. O tempo de perfusão variou de 112 a 285 minutos (média: 150 minutos; DP = 34). Em todos os casos, realizou-se a clássica técnica do transplante cardíaco ortotópico(4). Obteve-se a proteção miocárdica do coração do doador pela infusão de $1.000 \mathrm{ml}$ de solução cardioplégica St. Thomas e imersão em solução salina a $4^{\circ}$ C. O tempo de isquemia do enxerto variou de 60 a 180 minutos (média: 91 minutos; $\mathrm{DP}=21$ ).

A imunossupressão foi realizada pelo esquema tríplice composto de ciclosporina, azatioprina e corticóide na grande maioria dos pacientes. Nos últimos três casos, a azatioprina foi substituída pelo micofenolato de mofetil. Nos primeiros 10 doentes, a imunossupressão foi induzida pela globulina antilinfocitária. A ciclosporinemia foi periodicamente medida pelo método da radioimunoensaio. O diagnóstico definitivo de episódios de rejeição foi sempre estabelecido por estudo histológico de biópsias endomiocárdicas, seguindo os critérios classicamente estabelecidos (5)

\section{Análise Estatística}

$\mathrm{Na}$ análise dos resultados foram aplicados 0 teste de Mann-Whitney e o teste exato de Fisher. Em cada teste, adotou-se o nível de significância de $5 \%$. Para a mediana do tempo de sobrevivência, o nível de confiança foi de $95 \%$. A curva de sobrevida actuarial foi construída pelo método não-paramétrico de Kaplan-Meyer.

\section{RESULTADOS}

\section{Mortalidade Hospitalar}

Ocorreram 7 (20\%) óbitos na fase de internação hospitalar (Tabela 4). Um paciente (caso 14) faleceu na sala de operações em decorrência de coagulopatia. Três doentes (casos 7, 10 e 18) morreram devido à lesão neurológica, sendo que, em um deles, essa complicação decorreu de falha mecânica do oxigenador. Uma paciente (caso 5 ) faleceu no $28^{\circ}$ dia em conseqüência de miocardite por toxoplasma. Ela havia sido operada em falência múltipla de órgãos, com volumosa ascite e com grande escara sacral que exigiu enxerto de pele na mesma ocasião do transplante. No pós-operatório imediato, foi necessário reoperação para fechamento de fístula peritôneo-pericárdica através da qual o líquido ascítico encheu o saco pericárdico. Os outros óbitos decorreram de insuficiência renal aguda (caso 16) e parada cardíaca súbita (caso 30 ).

Não foi possível estabelecer correlação estatística entre esses óbitos e operações prévias, tempo de perfusão e tempo de isquemia do enxerto.

\section{Complicações Pós-operatórias}

Além dos pacientes que faleceram, 13 (37,1\%) outros apresentaram complicações durante o período de internação hospitalar (Tabela 2). As complicações mais freqüentes foram rejeição (5 casos), candidíase oral (4 casos), insuficiência renal que exigiu hemodiálise (4 casos) e sangramento, necessitando exploração cirúrgica (3 casos). Outras complicações ocorridas, em um caso cada, incluíram: convulsão, bloqueio $\mathrm{A}-\mathrm{V}$ total permanente, síndrome de baixo débito cardíaco que exigiu contrapulsação com balão intra-aórtico, lesão neurológica com déficit permanente, úlcera gástrica perfurada e escara sacral, estas últimas tratadas cirurgicamente.

\section{Pós-operatório Tardio}

A Tabela 3 agrupa os principais dados do pósoperatório tardio dos 28 pacientes que receberam alta hospitalar após o transplante cardíaco.

O tempo de evolução variou de 1 a 68 meses, com mediana igual a 31,2 meses (IC 95\%: 13 a 49 meses).

Vinte e três $(82,1 \%)$ dos 28 sobreviventes apresentaram algum tipo de complicação no decorrer desse período de observação. Doze pacientes apresentaram 24 episódios de rejeição aguda, que exigiu pulsoterapia, e 16 tiveram um total de 24 infecções. Outras compli- 
Moraes Neto F, Tenório D, Gomes C A, Tenório E, Hazin S, Magalhães M, Moraes C R - Transplante cardíaco: a experiência do Instituto do Coração de Pernambuco com 35 casos. Rev Bras Cir Cardiovasc 2001; 16(2): 152-9.

TABELA 2

\begin{tabular}{|c|c|c|c|}
\hline \multicolumn{4}{|c|}{ RESULTADOS IMEDIATOS } \\
\hline CASO & COMPLICAÇÕES & RESULTADO & CAUSA DO ÓBITO \\
\hline 1 & Reoperação sangramento/Rejeição (7º/22º PO) & Bom & -- \\
\hline 2 & Candidíase oral/Convulsão/Rejeição (7º PO) & Bom & -- \\
\hline 3 & IRA/Candidíase/Afta gigante & Bom & -- \\
\hline 4 & - & Bom & -- \\
\hline 5 & Fístula peritôneo-pericárdica/Rejeição (23ํPO) & Óbito $\left(28^{\circ} \mathrm{PO}\right)$ & Miocardite por toxoplasma \\
\hline 6 & Reoperação por sangramento/Afta gigante & Bom & -- \\
\hline 7 & Lesão neurológica & Óbito (9 PO) & Lesão neurológica \\
\hline 8 & Reoperação por sangramento/Candidíase/Rejeição (24ํPO) & Bom & - \\
\hline 9 & - & Bom & -- \\
\hline 10 & Lesão neurológica (falha do oxigenador) & Öbito (1ํ PO) & Lesão neurológica \\
\hline 11 & - & Bom & -- \\
\hline 12 & BAVT (Marcapasso)/Fístula pericárdio-cutânea & Bom & -- \\
\hline 13 & Lesão neurológica /Rejeição ( $8^{\circ} / 22^{\circ}$ PO) & Bom & -- \\
\hline 14 & - & Óbito (SO) & Coagulopatia \\
\hline 15 & Afta gigante/Candidíase esofágica e oral & Bom & -- \\
\hline 16 & IRA & Óbito (3ํㅜ) & IRA \\
\hline 17 & IRA/SBDC (BIA) & Bom & -- \\
\hline 18 & Lesão Neurológica & Óbito (7ํㅜ) & Lesão neurológica \\
\hline 19 & - & Bom & -- \\
\hline 20 & - & Bom & -- \\
\hline 21 & - & Bom & -- \\
\hline 22 & Rejeição (22 PO) & Bom & -- \\
\hline 23 & - & Bom & -- \\
\hline 24 & - & Bom & -- \\
\hline 25 & - & Bom & -- \\
\hline 26 & IRA/Úlcera gástrica perfurada (laparatomia) & Bom & -- \\
\hline 27 & IRA & Bom & -- \\
\hline 28 & Escara (enxerto de pele) & Bom & -- \\
\hline 29 & - & Bom & -- \\
\hline 30 & Bradicardia e parada cardíaca irreversível & Óbito (1ํPO) & Parada cardíaca \\
\hline 31 & - & Bom & - \\
\hline 32 & - & Bom & -- \\
\hline 33 & - & Bom & -- \\
\hline 34 & - & Bom & 一- \\
\hline 35 & - & Bom & -- \\
\hline
\end{tabular}

IRA = Insuficiência Renal Aguda; BAVT = Bloqueio A-V Total; SBDC = Síndrome do Baixo Débito; BIA = Balão Intra-aórtico.

cações incluíram o desenvolvimento de hipertensão arterial sistêmica (7 casos), de insuficiência renal crônica (4 casos), de diabete melito (3 casos) e episódios de hemorragia digestiva por sangramento de úlcera gástrica ou de pólipos intestinais.

Ocorreram 14 óbitos tardios em decorrência de rejeição (4 casos), infecção (4 casos), doença arterial coronária (2 casos), insuficiência renal crônica ( 2 casos) e morte súbita ( 2 casos). Dos 4 pacientes que faleceram por rejeição, 3 haviam abandonado o tratamento, e 2 deles haviam retornado ao hábito do alcoolismo. Por outro lado, dos 4 doentes que faleceram por infecção, em 2 a causa foi dengue.

\section{Curva Actuarial de Sobrevida}

A curva actuarial de sobrevida (Gráfico 1), incluindo a mortalidade operatória, mostra sobrevivência do receptor no $1^{\circ}, 2^{\circ}, 3^{\circ}, 4^{\circ}$ e $5^{\circ}$ anos, respectivamente de $70,3 \%, 66,9 \%, 46,5 \%, 36,2 \%$ e $30,1 \%$ 
Moraes Neto F, Tenório D, Gomes C A, Tenório E, Hazin S, Magalhães M, Moraes C R - Transplante cardíaco: a experiência do Instituto do Coração de Pernambuco com 35 casos. Rev Bras Cir Cardiovasc 2001; 16(2): 152-9.

TABELA 3

RESULTADOSTARDIOS

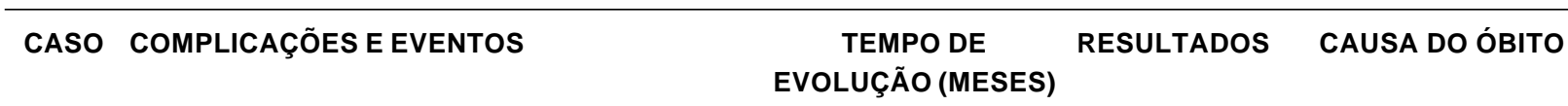

\begin{tabular}{|c|c|c|c|c|}
\hline 1 & HAS/Diabetes/Rejeição (3/36ํmeses) & 38 & Óbito & Doença arterial coronária \\
\hline 2 & $\begin{array}{l}\left.\text { Infecção respiratória ( } 3^{\circ} \text { mês }\right) / \text { Candidíase oral } \\
\left.\text { (4ํำ mês) /Rejeição ( } 2^{\circ} / 71^{\circ} \text { meses }\right)\end{array}$ & 7 & Óbito & Rejeição \\
\hline 3 & 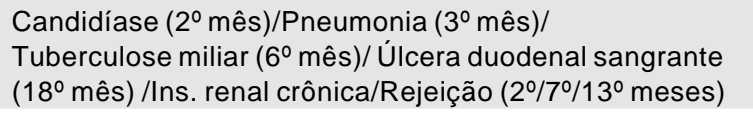 & 68 & Óbito & Morte súbita \\
\hline 4 & Diabetes/Rejeição ( $1^{\circ} / 18^{\circ}$ meses)/ Meningite (20ํำs) & 20 & Óbito & Meningite pneumocócica \\
\hline 6 & $\begin{array}{l}\left.\text { Infecção urinária e sepsis ( } 7^{\circ} \text { mês }\right) / \\
\left.\left.\text { Pneumonia ( } 8^{\circ} \text { mês }\right) / \text { Estreptococcia MID ( } 35^{\circ} \text { mês }\right) / \\
\left.\text { HAS/Rejeição ( } 2^{\circ} / 9^{\circ} / 48^{\circ} \text { meses }\right) / \text { Alcoolismo/ } \\
\text { Abandono do tratamento }\end{array}$ & 52 & Óbito & Rejeição \\
\hline 8 & $\begin{array}{l}\left.\text { Infeccão urinária (18ํㅡês)/ Diabetes ( } 7^{\circ} \text { mês }\right) / \\
\left.\text { Ins. renal crônica/Rejeição ( } 5^{\circ} \text { mês }\right) / \text { Alcoolismo }\end{array}$ & 35 & Óbito & Ins. Renal \\
\hline 9 & Insuficiência renal crônica/Erisipela ( $3^{\circ}$ mês) & 22 & Óbito & Ins. Renal \\
\hline 11 & $\begin{array}{l}\text { Infecção intestinal ( } 8^{\circ} \text { mês)/Abandono do tratamento / } \\
\text { Rejeição aguda ( } 22^{\circ} \text { mês) }\end{array}$ & 22 & Óbito & Rejeição \\
\hline 12 & HAS/Rejeição (3/8ำ meses) & 24 & Óbito & Morte súbita \\
\hline 13 & HAS/Hematêmese (20ำ mês)/ Depressão & 60 & Bom & -- \\
\hline 15 & 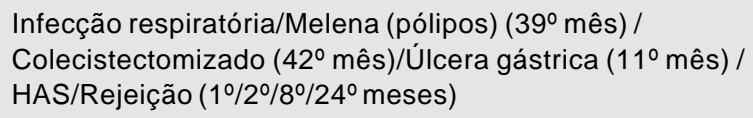 & 62 & Óbito & Doença arterial coronária \\
\hline 17 & - & 55 & Bom & - \\
\hline 19 & Fibrilação atrial/Rejeição (26ํㅡês) & 51 & Bom & -- \\
\hline 20 & - & 50 & Bom & -- \\
\hline 21 & Dengue (IRA/Hemorragia digestiva) ( $3^{\circ}$ mês) & 7 & Óbito & Dengue \\
\hline 22 & $\begin{array}{l}\text { Infecção respiratória ( } 23^{\circ} \text { mês)/Rejeição ( } 2^{\circ} / 4^{\circ} \text { - meses)/ } \\
\text { Alcoolismo/Otite/Sinusite ( } 7^{\circ} \text { mês)/ Abandono do tratamento }\end{array}$ & 31 & Óbito & Rejeição \\
\hline 23 & Dengue (IRA/Hemorragia digestiva) ( $7^{\circ}$ mês $)$ & 9 & Óbito & Dengue \\
\hline 24 & HAS/Obesidade & 31 & Bom & 一- \\
\hline 25 & Encefalite por toxoplasma ( $1^{\circ}$ mês) & 31 & Bom & - \\
\hline 26 & $\begin{array}{l}\left.\text { Infecção respiratória ( } 4^{\circ} \text { mês }\right) / \text { Ins. renal crônica } \\
\text { Meningite }\left(7^{\circ} \text { mês }\right) / \text { tuberculose }\left(7^{\circ} \text { mês }\right)\end{array}$ & 13 & Óbito & Meningite/tuberculose \\
\hline 27 & HAS & 26 & Bom & - \\
\hline 28 & Infecção respiratória (4ํmês)/obesidade & 18 & Bom & 一- \\
\hline 29 & Rejeição (9º mês) & 16 & Bom & - \\
\hline 31 & - & 10 & Bom & - \\
\hline 32 & - & 8 & Bom & - \\
\hline 33 & Herpes zoster ( $3^{\circ}$ mês) & 8 & Bom & -- \\
\hline 34 & Herpes zoster ( $2^{\circ}$ mês) & 6 & Bom & 一- \\
\hline 35 & - & 1 & Bom & - \\
\hline
\end{tabular}

HAS = Hipertensão Arterial Sistêmica; IRA = Insuficiência Renal Crônica; MID = Membro Inferior Direito 
Moraes Neto F, Tenório D, Gomes C A, Tenório E, Hazin S, Magalhães M, Moraes C R - Transplante cardíaco: a experiência do Instituto do Coração de Pernambuco com 35 casos. Rev Bras Cir Cardiovasc 2001; 16(2): 152-9.

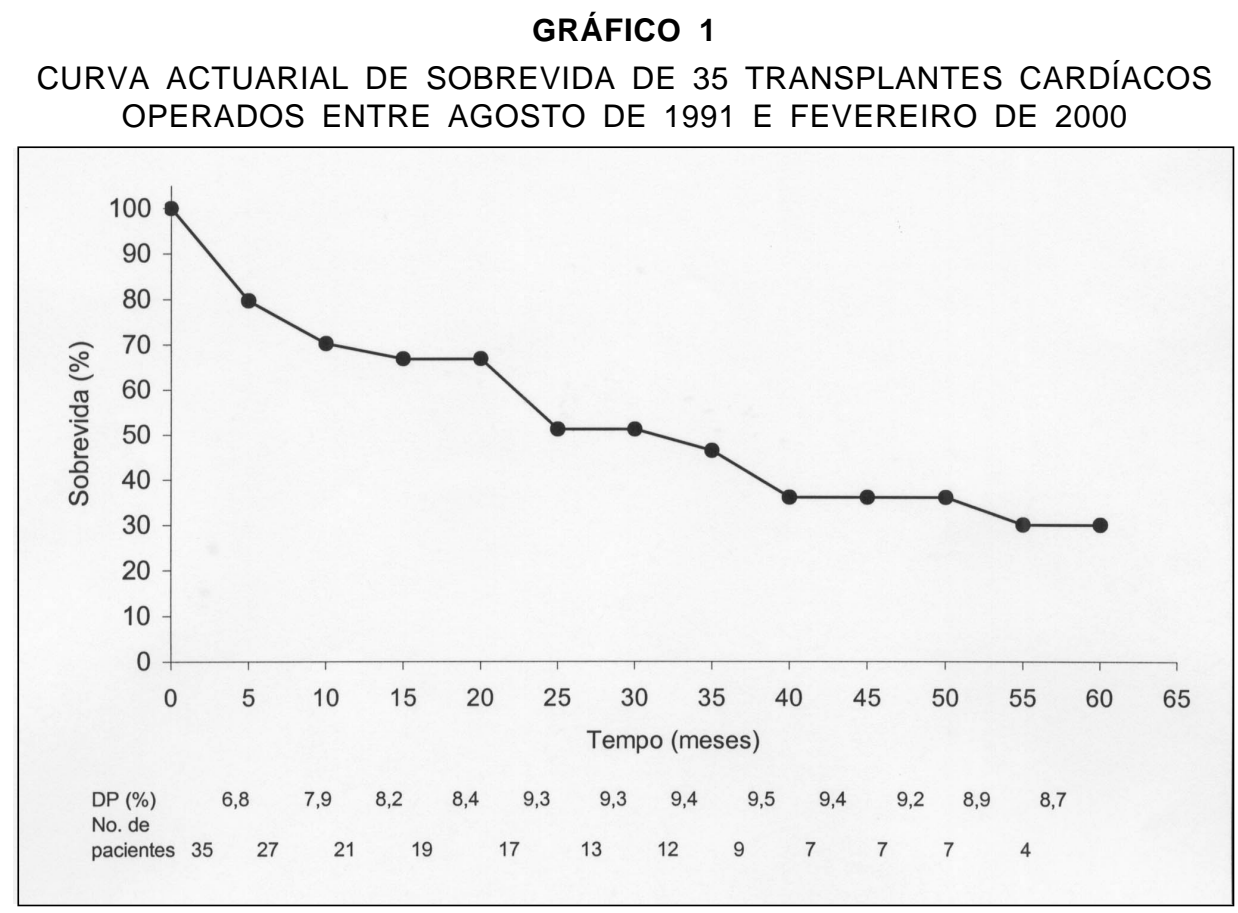

\section{COMENTÁRIOS}

No Brasil, onde a cirurgia cardíaca é muito desenvolvida, o número de centros que realizam rotineiramente transplantes cardíacos e o número anual de transplantes estão aquém do desejado ${ }^{(1)}$. Vários fatores contribuem para essa situação, entre eles a escassez de doadores, dificuldades na organização do sistema público de saúde e problemas socioeconômicos. Em 1991, estabelecemos no Instituto do Coração do Real Hospital Português de Beneficência em Pernambuco um programa de transplante cardíaco. Desde então, mais de 200 doentes foram avaliados no ambulatório especializado, e 65 preencheram os critérios de indicação de transplante cardíaco. Entretanto, apenas 35 transplantes foram realizados nesse período. Os outros ou faleceram antes do procedimento ou continuam em lista de espera, o que demonstra a dificuldade desse tipo de tratamento em nosso meio.

A distribuição por sexo e idade de nossos pacientes corresponde à experiência de outros centros ${ }^{(6)}: 82,9 \%$ eram homens e a média de idade foi de 46,9 anos, sendo que $16(45,7 \%)$ tinham mais de 50 anos. Com relação às doenças que levaram à indicação do transplante, nossos dados também correspondem à experiência internacional, observando-se predomínio das miocardiopatias isquêmica e dilatada idiopática ${ }^{(6)}$.
Com relação ao ato cirúrgico, utilizamos em todos os casos a técnica clássica de transplante ortotópico de LOWER \& SHUMWAY (4) que, em nossa pequena experiência, mostrou-se simples e efetiva. Atualmente, na experiência internacional, cerca de $80 \%$ dos transplantes são realizados com captação à distância (6). Entretanto, tal como vem ocorrendo em outros centros de nosso país ${ }^{(1,7)}$, a maioria $(94,2 \%)$ dos nossos transplantes foi realizada com a coleta do órgão em sala contígua. Essa prática oferece maior conforto e segurança às equipes, pois diminui o tempo de isquemia do enxerto que, em nossa série, foi em média de $91 \pm 21$ minutos.

A mortalidade imediata em nossa série foi elevada, mas é comparável à relatada em outras séries brasileiras $(1,7)$. Dois fatores, em nossa opinião, podem explicar esse fato: a natural curva de aprendizado que todos os Serviços experimentam e a precária situação clínica pré-operatória da maioria dos doentes. Seis dos 7 óbitos imediatos ocorreram entre os primeiros 18 casos operados, enquanto, nos últimos 17 transplantes, houve apenas uma morte. Por outro lado, a grande maioria dos doentes estava em fase final de insuficiência cardíaca, necessitando inotrópicos (3 se encontravam moribundos ao serem levados à sala de operações). Outro dado importante é que $17(48,5 \%$ dos casos) já haviam sido submetidos a pelo menos uma operação cardíaca previamente. 
Moraes Neto F, Tenório D, Gomes C A, Tenório E, Hazin S, Magalhães M, Moraes C R - Transplante cardíaco: a experiência do Instituto do Coração de Pernambuco com 35 casos. Rev Bras Cir Cardiovasc 2001; 16(2): 152-9.

A causa de óbito imediato mais freqüente (3 casos) foi lesão neurológica, complicação que tende a diminuir com o aumento da experiência (7). Um paciente, já operado anteriormente, e cujo descolamento do coração foi tecnicamente difícil, faleceu na sala de operações devido à coagulopatia. Os outros óbitos decorreram de miocardite por toxoplasma, insuficiência renal aguda e parada cardíaca súbita, causas freqüentemente registradas na literatura $(6,7)$.

No período de internação hospitalar, 13 pacientes apresentaram complicações não fatais dentro do contexto esperado e relatado na literatura, após transplante cardíaco ${ }^{(7,8)}$. Deve ser ressaltado que, excetuando 4 pacientes que exibiram candidíase oral, não ocorreram complicações infecciosas no pós-operatório imediato. Episódios de rejeição foram diagnosticados em 5 casos.

$\mathrm{Na}$ evolução tardia de nossos doentes, encontra-se provavelmente a grande diferença entre a nossa experiência com o transplante cardíaco e a experiência internacional e a de outros centros brasileiros ${ }^{(6,7)}$. Vinte e três $(82,1 \%)$ dos 28 sobreviventes apresentaram complicações ao longo de um período de observação que variou de 1 a 68 meses, com mediana igual a 31,2 meses, predominando 24 episódios de rejeição aguda, ocorridos em 12 doentes e 24 infecções, apresentadas por 16 pacientes. Ocorre que muitas dessas complicações, já freqüentes após transplante, e que contribuíram para a elevada mortalidade tardia, foram seguramente provocadas por falta de aderência do paciente ao tratamento e aos cuidados pós-transplante. Três pacientes abandonaram o tratamento imunossupressor, e 2 retornaram ao hábito do alcoolismo. Ademais, 2 outros doentes contraíram dengue, infecção endêmica e muito freqüente em nosso meio. Apesar de benigna, na maioria dos casos, a dengue tem-se mostrado fatal quando atinge pessoas imunodeprimidas.

A mortalidade tardia ocorreu em 14 casos, e além de rejeição (4 casos) e de infecção (4 casos), outras causas de óbito incluíram doença arterial coronária (2 casos), insuficiência renal crônica ( 2 casos) e morte súbita (2 casos). Quando construída a curva actuarial de sobrevivência, verificamos que a perspectiva de sobrevida de um ano $(70 \%)$ e de 5 anos (30\%) difere substancialmente da experiência internacional e da brasileira $(1,6,7)$ em decorrência das limitações sociais da maioria da nossa população de receptores.

Baseados na experiência descrita no presente trabalho, é lícito concluir ser possível a manutenção de um programa de transplante cardíaco em nosso meio, devendo-se, contudo, aguardar resultados menos favoráveis do que os obtidos em regiões economicamente mais desenvolvidas em face das limitações sociais da população de receptores.

RBCCV 44205-542

Moraes Neto F, Tenório D, Gomes C A, Tenório E, Hazin S, Magalhães M, Moraes C R - Heart transplant: the experience of the Heart Institute of Pernambuco with 35 cases. Rev Bras Cir Cardiovasc 2001; 16(2): 152-9.

ABSTRACT: Material and Methods: From August/ 1991 to February/ 2000, 35 orthotopic heart transplants were performed at the Heart Institute of Pernambuco. There were 29 male and 6 female patients ranging in age from 15 to 69 years (mean: $46.9 \mathrm{yr}$.). Recipient diagnoses included ischemic cardiomyopathy in 18, idiopatic dilated cardiomyopathy in 13 , mixomatous disease in 2 , rheumatic valvar disease in 1 and Chagas' disease in 1. All were in the final stage of heart failure (functional class III or IV of the New York Heart Association) and the ejection fraction ranged from 16 to 27\% (mean: $20.9 \pm 2.9$ ). Seventeen $(48.5 \%)$ patients had had previous cardiac operations. The classical surgical technique described by Lower and Shumway, in 1960, was used in all cases. Mean graft ischemic time was $91 \pm 21$ minutes (ranged from 60 to 180 minutes).

Results: There were $7(20 \%)$ deaths in the early postoperative period. Non-fatal postoperative complications occurred in other 13 patients including 5 episodes of rejection. Mean follow-up period of the 28 survivors was 31.2 months (ranged from 1 to $68 \mathrm{mo}$.) There were 14 late deaths owing to rejection (4), infection (4), graft coronary artery disease (2), chronic renal failure (2) and sudden death (2). The actuarial survival estimated is $70 \%$ at 1 year and $30 \%$ at 5 years.

Conclusion: It is concluded that cardiac transplantation program is feasible in our community but poorer results in comparison to international experience should be expected owing to social problems of the recipient population.

DESCRIPTORS: Heart transplantation. Heart diseases, surgery. 


\section{REFERÊNCIAS BIBLIOGRÁFICAS}

1 Stolf N A - Transplante cardíaco humano no Brasil. Arq Bras Cardiol 1994; 63: 247-50.

2 Moraes C R, Rodrigues J V, Tenório D et al. - Transplante de coração: experiência inicial no Instituto do Coração de Pernambuco. Arq Bras Cardiol 1992; 59: 47-9.

3 Jamieson S W, Oyer P E, Reitz B A et al. - Cardiac transplantation at Stanford. Heart Transplant 1983; 1: 86-91.

4 Lower R R \& Shumway N E - Studies on orthotopic homotransplantation of the canine heart. Surg Forum 1960; 11: 18-9.

5 Billingham M E, Cary N R, Hammond M E et al. - A working formulation for the standardization of nomenclature in the diagnosis of heart and lung rejection: Heart Rejection Study Group. J Heart Transplant 1990; 9: 587-93.

6 Hosenpud J D, Bennett L E, Keck B M, Fiol B, Novick $\mathrm{R} J$ - The Registry of the International Society for Heart and Lung Transplantation: fourteenth official report -1997. J Heart Lung Transplant 1997; 16: 691-712.

7 Branco J N R, Teles C A, Aguiar L F et al. - Transplante cardíaco ortotópico: experiência da Universidade Federal de São Paulo. Rev Bras Cir Cardiovasc 1998; 13: 285-94.

8 Fiorelli A I \& Stolf N A G - Cuidados no pós-operatório de transplante cardíaco. Rev Bras Cir Cardiovasc 1996; 11: 30-8. 